\title{
Introduction: Advances in E-business engineering
}

\author{
Ling Li
}

Published online: 12 May 2011

(C) Springer Science+Business Media, LLC 2011

E-business is more than just e-commerce. It is one of the most challenging areas for industry and research communities. E-business has evolved from business-to-business, business-to-customer, customer-to-business, customer-tocustomer, and business-to-government systems to the integrated and collaborative business services among various information systems and e-marketplaces. In this evolving process, integrated e-business systems and their related supporting platforms have to be rapidly designed and developed in order to meet different requirements. A variety of e-business engineering paradigms and technologies have been developed to tackle these challenges. There are many research issues needed to be addressed. These issues include heterogeneous services integration, disparate e-business functions collaboration, semantic level e-business messaging, etc. Today, not only large companies, but also medium or small-sized companies are learning that e-business is a required component of doing business. As a result, there is a growing demand for insights into challenges, issues, and solutions related to the design, implementation, and management of e-business systems.

To respond to the market needs from both academic researchers and practitioners, and to communicate research results on e-business engineering, a number of related conferences organized by the IFIP TC8 WG8.9, IEEE Computer Society Technical Committee on E-Commerce, and IEEE SMC Society Technical Committee on Enterprise Information Systems have been held in the past years. Such conferences include IEEE International Conference on e-Business Engineering, IFIP International Conference

L. Li $(\bowtie)$

Department of Information Technology and Decision Sciences, Old Dominion University, Norfolk, VA 23529, USA

e-mail: 1li@odu.edu on Research and Practical Issues of Enterprise Information Systems (Confenis), IEEE SMC International Conference Special Session on Enterprise Information Systems, GCIS, and WCSE. These conferences plus workshops have provided an international forum for researchers in academia and industry to present their most recent findings in e-business engineering.

This special issue of Information Technology and Management presents expanded versions of 12 papers from the above-mentioned conferences held recently. The purpose of this special issue is to report on the state-of-the-art of, and emerging trends in, research and practice in e-business engineering. To prepare for this issue, all authors were asked to respond to two rounds of peer review. Each paper emphasizes the importance of e-business engineering from a unique perspective.

In the paper by Academician Shoubo Xu of the Chinese Academy of Engineering, a new concept of management is proposed. The author indicates that "to manage" means to lead, to plan, to organize and to control; and "to reason", means to understand the law of the development of objects being managed. This new concept is an integration of the two elements of "managing" and "reasoning". As such, management is to study the contradictory relationship between "managing" and "reasoning", and consider how such a relationship develops and changes. The paper discusses its broad applications including information industry such as e-business.

Qian, Jin and Fang of the Institute of Software, Chinese Academy of Sciences, in their paper entitled "Heuristic algorithms for effective broker deployment", proposes a model for the broker deployment problem, and presents two heuristic algorithms for the multi-object optimization of broker deployment. Experiments are conducted to demonstrate the effectiveness of the proposed algorithms. 
The paper by Liu et al. of Tsinghua University identifies main issues in distributed data mining (DDM) systems for e-business and classifies modern DDM systems into three classes. The authors propose a novel DDM model named DRHPDM (Data source Relevance-based Hierarchical Parallel Distributed data mining Model).

In the paper entitled "Cohesion and coupling metrics for ontology modules", Oh, Yeom and Ahn of the Seoul National University, Korea, have proposed novel metrics to measure ontology modularity. The proposed metrics provide more detailed support in considering the different types of relationships between classes in ontology modules. The proposed metrics have been validated using wellknown verification frameworks and empirical experiments. The results offer ontology engineers valuable criteria for evaluating ontology modules and helping ontology users select qualifying ontology modules.

Wang and Wu of the University of Delaware (USA) and Old Dominion University (USA), in their paper entitled "Supporting process design for e-business via an integrated process repository", have proposed a collaborative approach grounded in knowledge management theory to integrate multiple process reference models to better support process design in e-business. A prototype system has been developed and user experiments have been conducted to evaluate the system.

Wang et al. from Beijing University of Aeronautics and Astronautics, in their paper entitled "A decision support system for substage-zoning filling design of rock-fill dams based on particle swarm optimization", have proposed a particle swarm optimization based decision support system for rock-fill dams. The system has been employed in a water conservancy and hydropower project which shows that the system is able to provide quality decision support and facilitate the rock-fill dam construction effectively.

In the paper entitled "Measuring virtual wealth in virtual worlds", Guo and Gong of the University of Macau, presents pioneering research on measuring virtual wealth in an open virtual world for diagnosing the health of virtual worlds. It proved the existence of an open virtual world by proving the existence of a free choice of virtual currencies for virtual goods between distinct virtual worlds. The proposed measuring scheme and matrix help virtual world participants perceive the possible risks of the participated virtual worlds in advance to make better decisions on virtual business.

Xie et al. of Wuhan University of Technology in the paper entitled "Research on the group decision-making about emergency event based on network technology", have detailed the mechanism of group decision-making about emergency event based on network technology and agile Delphi Method.
In the paper entitled "An enhanced model framework of personalized material flow services", Hou et al. of Beijing Jiaotong University, Vienna University of Economics and Business (Austria), Vienna University of Technology (Austria), and Ferris State University (USA), have proposed an enhanced conceptual model for Personalized Material Flow Services (PMFS) for consumer behavior. E-business examples are used to analyze the elements such as virtual collector, information filter and material flow coordinator in the PMFS system.

In the paper entitled "An evidence-based scheme for web service selection", Wang et al. of Kun Shan University, National Chiao Tung University, and Coventry University (UK) have proposed a trust-based service selection model to estimate the degree of consumer trust in a particular service based on the consumers' direct experience and indirect recommendation of the service. In the proposed approach, the degree of consumer trust is estimated by extending Dempster-Shafer evidence reasoning theory to the reputation computation using consumers' direct experience and incorporating Jøsang's belief model for solving the trust transition problem in the indirect recommendation of the service.

Shan et al. of Beijing University of Aeronautics and Astronautics, in their paper entitled "Research on e-Government evaluation model based on the principal component analysis", with principal component analysis, have presented, validated and updated an evaluation model with 5 dimensions based on socio-technical model and stakeholder theory, which captures the multidimensional and interdependent nature of e-government system. The findings provide several important implications for e-government research and practice.

In the paper entitled "Constructing a decision support system for management of employee turnover risk", Wang et al. have proposed a decision support system for employee turnover risk management using message processing mechanisms and software combination technology and system integration.

As the organizers of the above-mentioned conferences, we are delighted to share this sampling of the conferences with the readership of Information Technology and Management. We hope that this special issue will serve our Information Technology and Management readers as an avenue to gain a new perspective on e-business engineering. We would specially like to thank the Editors-in-Chief, Professor H. Pirkul and Professor V. S. Jacob, for their encouragement and guidance throughout this endeavor. We are also deeply grateful to the many individual reviewers who worked with us so diligently. Without their time, effort and support, this issue would never have come to be. 